\title{
Modelo de predicción de la vida útil de la edificación patrimonial a partir de la lógica difusa
}

\section{Prediction model of the useful life of a heritage building from fuzzy logic}

\author{
$\underline{\text { J. M. Macías-Bernal }}^{(*)}$, J. M. Calama-Rodríguez ${ }^{(*)}$, M. J. Chávez-de Diego
}

\section{RESUMEN}

Buscamos un procedimiento que permita determinar y valorar los factores que inciden sobre la vulnerabilidad y riesgos que afectan al edificio y validar un modelo para poder calcular su vida útil. Tendríamos así un método de predicción que resultaría de mucha utilidad en los procesos de mantenimiento y conservación del patrimonio edificado. El problema es que al analizar las diversas metodologías que actualmente se utilizan para la predicción de hechos en el campo de la conservación del patrimonio, nos encontramos con que tales métodos se hallan en el umbral de la incertidumbre. Por esta causa, hemos optado por trabajar con la teoría de conjuntos difusos (Fuzzy set), como herramienta más adecuada. Al modelo de predicción generado con base matemática, lo hemos denominado FBSL (Fuzzy building service life) y ofrece unos resultados satisfactorios, a la hora predecir la vida útil del edificio.

Palabras clave: Conservación; patrimonio; lógica difusa; vida útil; durabilidad.

\section{SUMMARY}

We seek a procedure to identify and assess the factors that affect the vulnerability and risks affecting the building and validate a model to calculate service life. We would thus have a prediction method that would be very useful in the process of maintenance and conservation of the built heritage. The problem is that in analyzing the various methods currently used to predict events in the field of heritage conservation, we find that such methods are at the threshold of uncertainty. For this reason, we have chosen to work with the theory of fuzzy sets (fuzzy set), as most appropriate tool. When generated prediction model based mathematics, what we have called FBSL (Fuzzy building service life) and provide satisfactory results, when predicting the building service life.

Keywords: Conservation; heritage; fuzzy logic; service life; durability.

(*) Universidad de Sevilla (España).

Persona de contacto/Corresponding author: jmmacias@us.es (J.M. Macías-Bernal)

Cómo citar este artículo/Citation: Macías-Bernal, J. M., Calama-Rodríguez, J. M., Chávez-de Diego, M. J. (2014). Modelo de predicción de la vida útil de la edificación patrimonial a partir de la lógica difusa. Informes de la Construcción, 66(533): eoo6, doi: http://dx.doi.org/10.3989/ic.12.107.

Licencia / License: Salvo indicación contraria, todos los contenidos de la edición electrónica de Informes de la Construcción se distribuyen bajo una licencia de uso y distribución Creative Commons Reconocimiento no Comercial 3.o. España (cc-by-nc). 


\section{INTRODUCCIÓN}

La gestión de la conservación del patrimonio se puede abordar desde múltiples enfoques, en nuestro caso hemos considerado de interés el poder conocer, con alguna certeza, la durabilidad en el tiempo del patrimonio edificado, como criterio a considerar para la toma de decisiones relacionadas con la programación y planificación de los distintos procesos de intervención.

Además, para predecir el tiempo de vida útil que puede tener un edificio hay que analizar los riesgos a los que se verá sometido durante su uso y cómo afronta o previene los posibles daños que estos riesgos le pueden causar.

Por otro lado, analizar la vulnerabilidad intrínseca de una edificación y ponerla en relación con los riesgos a la que está sometida, genera una gran cantidad de variables que deben ser procesadas y ordenadas de forma objetiva, por lo que parece oportuno plantear un modelo de análisis que nos permita predecir cuál va a ser la evolución y el estado de conservación en el tiempo de una edificación, a partir de un conjunto de variables aceptado por un grupo de expertos.

La necesidad de plantearnos el diseñar un modelo de predicción de la vida útil de un edificio, nos surge cuando analizamos un número elevado de bienes inmuebles, y que no están estudiados de forma conjunta. No es posible actuar simultáneamente en todos, ya que generalmente contamos con recursos limitados y, por tanto, debemos asignarlos de forma eficiente. Sabemos, que si realizamos una fotografía fija del estado de aptitud de una serie de edificios, comprobaríamos como en el futuro, cada uno de ellos habrá evolucionado de forma diferente. Todo esto se debe a la diversidad de factores de degradación a los que están sometidos los edificios, y la intensidad con que cada uno puede afectarle.

En edificación hay algunos modelos que ya utilizan el «diagnóstico», es decir: conocer el proceso, el origen, las causas, su evolución, síntomas y el estado actual, para predecir consecuencias. Es, por ejemplo el caso de la «Utilización de la Inteligencia Artificial en el diagnostico patológico de edificaciones de valor patrimonial» (1). Se trata de un modelo que al igual que en nuestro caso, usa un Sistema Experto planteado a partir de la Inteligencia Artificial y los Sistemas Basados en el Conocimiento, para identificar y diagnosticar patologías. Aunque, en este caso, únicamente se hace referencia a aquellas que presentan los materiales de Piedra Natural y Cerámicos, que se utilizan en construcción y nunca a los edificios en forma global. No obstante, tiene en común con nuestro sistema en que se crea un entorno informatizado que facilita la actualización e integración de datos relacionados con el deterioro de las edificaciones.

Por tanto, carecemos de una herramienta o modelo de predicción de la vida útil de cada edificio y tomamos las decisiones basándonos en el estado actual del inmueble. Normalmente no tenemos en cuenta la evolución del estado de conservación a lo largo de los años. No existen ni criterios, ni modelos que analicen estas situaciones, y consecuentemente se toman decisiones sin considerar como afecta el paso del tiempo a un conjunto de edificios diferentes, con los errores que pudieran conllevar.

Por ello, el diseño de una herramienta científico-técnica que le facilite la toma de decisiones con respecto a las estrategias que debe considerar para gestionar las acciones de su mantenimiento y restauración, nos parece de gran utilidad.

El primer paso en este sentido, es establecer las variables que intervienen en la vulnerabilidad y el riesgo, así como las relaciones que interactúan entre las mismas. Sin embargo, nos parece prácticamente imposible conseguir la unicidad de criterio si actuamos únicamente a partir de los datos y opiniones que puedan ofrecernos algunos expertos. Por ello, y con el fin de robustecer la fiabilidad y objetividad de las mismas, el modelo de análisis que hemos diseñado se sustenta sobre la teoría de conjuntos difusos o lógica difusa (también conocida como borrosa), planteada por Lofti Zadeh en 1965 (2), ya que se trata de una herramienta, capaz de relacionar matemáticamente un gran número de variables con alto grado de incertidumbre (3).

Partiendo de estas premisas, se plantea el presente trabajo de investigación enfocado al estudio de un conjunto de edificios que presentan características diferenciadas, pero que tienen en común el pertenecer a la Archidiócesis de Sevilla. Esto nos ha permitido estudiar edificios que no obedecen a ningún proceso de selección previo, sino que corresponden a un periodo cronológico de actuaciones profesionales que se han llevado a cabo en los mismos y cuyo objetivo común es: analizar su grado de aptitud actual y valorar las expectativas de vida útil a medio y largo plazo, con el fin de gestionar los recursos para su mantenimiento y restauración.

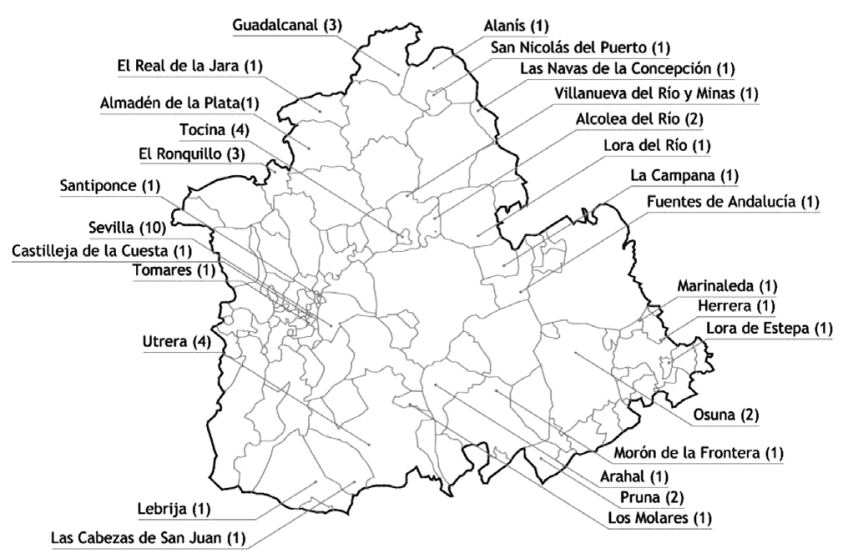

Figura 1. Mapa de distribución de inmuebles estudiados de la Archidiócesis de Sevilla.

El número de estos inmuebles, dispersos por toda la provincia de Sevilla (Figura 1), supera los setecientos edificios y aunque la actividad, en casi todos ellos, es de uso religioso, las características arquitectónicas y constructivas son muy diferentes. Además, tanto la fecha de construcción de cada uno de ellos, como las actuaciones de conservación, reconstrucción o rehabilitación llevadas a cabo en los mismos han sido múltiples y variadas, por lo que nos encontramos con gran número de factores que deberán ser considerados a la hora de diseñar un modelo de predicción.

Se estima, para el diseño del modelo, que todos estos edificios tienen una importancia similar dentro de la estructura social, cultural y patrimonial de la provincia. Cada uno de ellos requiere unas intervenciones muy particulares y adaptadas a cada caso, desde un punto de vista estrictamente técnico y económico. Esto hace que sea inviable la posibilidad de acometer tales trabajos de forma inmediata y simultánea. 
La propia estructura interna de funcionamiento de la Archidiócesis proporciona los criterios de prioridad de intervención, tanto con fines de mantenimiento como de su posible restauración, también hay que sumar los criterios de orden pastoral o de disponibilidad y aportaciones económicas de cada parroquia.

Por todo ello, un modelo de predicción como el que se propone, sustentado en el establecimiento de un criterio de análisis homogéneo, estamos seguros que podrá ser una herramienta de utilidad, en la toma de decisiones de los gestores y técnicos encargados del mantenimiento y restauración de este patrimonio.

\section{CONSIDERACIONES PREVIAS}

Antes de proceder al diseño de un modelo de predicción eficiente, es necesario analizar los factores de vulnerabilidad y riesgo a los que se encuentran sometidos los edificios.

Citemos entre otros documentos, la Carta del Riesgo de Italia (4) que se presenta como la primera experiencia para valorar, de forma global, los factores de degradación del Patrimonio Monumental. En la actualidad, sigue siendo una herramienta muy completa para abordar cuestiones de esta magnitud. No obstante, en nuestra opinión, aunque analiza distintos riesgos a los que puede estar sometido un edificio, utiliza la probabilidad estadística para sus determinaciones, lo que le proporciona un carácter demasiado aleatorio. Por otro lado, no aborda la noción de la vulnerabilidad (5), esto es: «la capacidad que el edificio tiene para soportar estos riesgos, teniendo como base su concepción arquitectónica y constructiva, así como su estado de conservación». Tampoco determina la vida útil de la edificación.

En segundo lugar citemos, la reciente, norma ISO 15686 Standards on Service Life Planing publicada en enero de 2011 (6), que puede ser un referente en cuanto al estudio y análisis de la vida útil de un edificio. Para su valoración plantea diversas metodologías de predicción sustentadas en datos de probabilidad estadística.

En nuestra opinión, las dos herramientas pueden ser mejoradas, ya que lo óptimo es conjugar ambas en un único modelo más completo que determinará de manera concreta el tiempo de vida útil del edificio, analizando la vulnerabilidad intrínseca del mismo y la pusiera en relación con los riesgos a los que está sometido. El problema es que todo este proceso genera una gran cantidad de variables que hay que ordenar y procesar de una forma objetiva. La praxis habitual resuelve esta incertidumbre recurriendo al concurso de expertos (7) sobre esta materia para que se pronuncien en tal sentido. El inconveniente está en que a veces la convergencia de criterios entre los expertos no es fácil. Por ello, nuestra aportación incorpora un modelo matemático que recoge este nivel de incertidumbre.

\section{OBJETIVOS DE LA PROPUESTA}

Hemos considerado básico para la definición del modelo en primer lugar, definir una serie de factores necesarios y suficientes para determinar la posible evolución en el tiempo del edificio. Una vez concretado el objetivo anterior, nos plan- teamos diseñar un modelo o sistema experto de predicción de la vida útil del conjunto de los edificios que vamos a inspeccionar, basado en los principios de la lógica difusa. El resultado de esta herramienta nos va a permitir establecer una calificación de carácter general, para cada uno de los cincuenta inmuebles, en base a la aplicación del modelo predictivo definido.

Así mismo El modelo de predicción de la vida útil que planteamos, hay que acotarlo bajo las siguientes premisas:

$1^{\mathrm{O}}$ Tanto los factores que vamos a utilizar, como las reglas que estableceremos entre ellos, se refieren a un conjunto de edificios con características propias, en cuanto a su uso religioso y en cuanto a su emplazamiento en diversos municipios de la provincia de Sevilla. Para aplicar este modelo a otros conjuntos edificatorios habría que realizar los ajustes oportunos de factores, funciones y reglas.

$2^{\mathrm{O}}$ El modelo se aplica a un conjunto de edificios y establece un orden de prelación entre ellos en cuanto a la vida útil estimada.

\section{METODOLOGÍA}

Podemos decir, de manera esquemática, que el desarrollo de la investigación se centra en diseñar un modelo que permita ordenar, según el criterio de vida útil, una serie de edificios patrimoniales, teniendo como base su vulnerabilidad a los riesgos a los que está sometido, con el fin de aportar datos cuantitativos y cualitativos a las acciones de gestión del patrimonio. Y para comprobar su validez, lo hemos aplicado a cincuenta edificios pertenecientes a la Archidiócesis de Sevilla ${ }^{1}$.

En este sentido el proceso metodológico para la creación del modelo, se ha estructurado en cuatro fases:

$1^{\text {a }}$ Determinar, según las fuentes existentes, los factores de vulnerabilidad y riesgo de mayor incidencia que pueden afectar a una edificación y que pueden influir en su durabilidad o vida útil.

$2^{\text {a }}$ Validar y ponderar, utilizando el concurso de un conjunto de expertos, los factores obtenidos en el paso anterior.

$3^{\text {a }}$ Diseñar un modelo experto, capaz de tratar los datos obtenidos en el concurso de los expertos, tomando como base la teoría de los conjuntos «borrosos o difusos», así como implementar el modelo con Xfuzzy_3.o.

$4^{\mathrm{a}}$ Tratar de manera interactiva los datos en cada uno de los cincuenta edificios y ordenarlos según su vida útil estimada.

\subsection{Fase $1^{\mathrm{a}}$ : Determinación de los factores:}

Como hemos adelantado, existen algunos métodos de análisis que determinan los factores de vulnerabilidad y riesgo. En este sentido nos hemos limitado a analizar diferentes trabajos y procedimientos de instituciones de prestigio, relacionados con la vida útil de los edificios. Se ha construido una matriz que recoge el conjunto de todos los factores con el fin de comprobar las debilidades y fortalezas de los mismos, para seleccionar de este modo los más adecuados. Para ello se ha hecho una revisión de los datos que aportan:

- El Plan Nacional de Catedrales (1991)

- La Ley de Ordenación de la Edificación (1999)

${ }^{1}$ Suponen el seis y medio por ciento del total que conserva la Archidiócesis de Sevilla. 
- El Método Rehabimed (2005)

- La Red de Conservación del Patrimonio (2006)

- El Código Técnico de la Edificación (2006)

- La Norma UNE sobre diagnóstico de edificios (2010) (8)

- La Norma ISO 15686 Sobre la determinación de la vida de servicio o vida útil del edifico (2011)

En la Tabla 1, se recogen las instituciones mencionadas, los factores propuestos por las mismas y, en la última fila, la síntesis de factores de nuestra propuesta.

Los factores de: «Protección contra el ruido», "Ahorro energético» $\mathrm{y}$ "Valor económico», no se han incluido en nuestra propuesta por considerar que en los documentos analizados se plantean con otras finalidades que nada o poco tienen que ver con la vida útil del edificio. El de «Tipo de edificio", no procede, ya que para la toma de datos a introducir en el modelo de análisis, se ha diseñado una ficha específica que permite valorar la exposición a la vulnerabilidad, desde un punto de vista arquitectónico y constructivo. Por último, hay que señalar que aunque el de la «Fecha de construcción», inicialmente no ha sido considerado, por haber sido matizado por alguno de los expertos, no se descarta realizar un estudio más exhaustivo que considere este aspecto.

\subsection{Fase $2^{a}$ : Validar y ponderar los factores.}

Como resultado, se han obtenido diecisiete factores determinantes, que son los que se han sometido a la valoración de quince expertos ${ }^{2}$. Calculando la media aritmética de la valoración de los expertos en una escala de cero a diez
(Cero indica nula incidencia del factor y diez incidencia total del factor propuesto). Obtenemos los resultados que se presentan en la Tabla 2. Hemos ordenado los factores en función de la importancia que han marcado los expertos.

\subsection{Fase $3^{\text {a }}$ : Diseñar el modelo experto.}

Sabemos que los procedimientos de modelos universales, basados en la lógica tradicional Aristotélica, son los que han hecho que el conocimiento científico avance en todos los campos del saber. En algunos aspectos el avance se detiene, porque no encuentra la respuesta adecuada, o mejor dicho, encuentra varias respuestas para una misma cuestión. Esta situación de paralización o estancamiento del avance del conocimiento, se agudiza cuando los problemas son más complejos, intervienen diversos factores que admiten diversas valoraciones, y además se aportan datos de diferentes magnitudes y disciplinas. No existe, entonces, una única respuesta verdadera o adecuada, incluso esa respuesta puede variar en el tiempo. El cerebro humano difiere en este sentido de la estricta lógica matemática, y basándose en el mayor número de datos e informaciones posibles, toma una decisión. No todos los humanos tomamos las mismas decisiones ante una misma situación. Ni siquiera una comunidad de expertos en una materia coinciden plenamente en el análisis de resultados.

Un edificio y su relación con el entorno es, sin duda, un sistema complejo de relaciones entre factores o variables (9). Cuando afrontamos una situación sobre la que tenemos un conocimiento impreciso y los datos que disponemos tienen

Tabla 1.

Factores de vulnerabilidad y riesgo.

\begin{tabular}{|c|c|c|c|c|c|c|c|c|c|c|c|c|c|c|c|c|c|c|c|c|c|c|c|c|}
\hline & \multicolumn{23}{|c|}{ FACTORES } \\
\hline & & 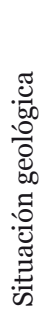 & 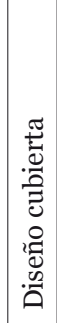 & 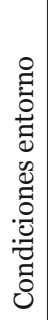 & 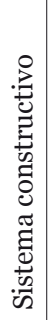 & 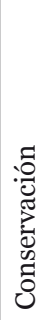 & 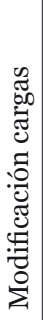 & 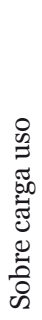 & 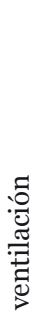 & 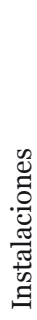 & 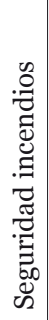 & 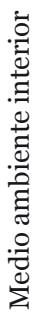 & 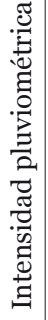 & 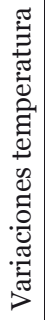 & 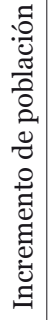 & 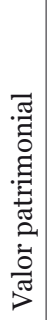 & 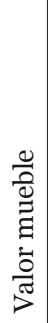 & 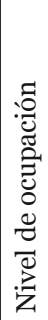 & 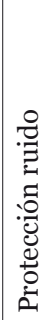 & 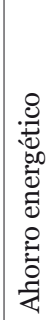 & 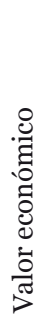 & 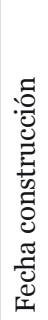 & 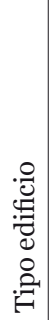 & 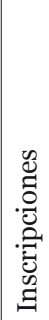 \\
\hline \multirow{8}{*}{ 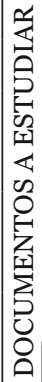 } & Ley 38/1999 & & & & & & & & & & & & & & & & & & & & & & & \\
\hline & CTE & & & & & & & & & & & & & & & & & & & & & & & \\
\hline & UNE $41805-3$ & & & & & & & & & & & & & & & & & & & & & & & \\
\hline & ISO 15686 & & & & & & & & & & & & & & & & & & & & & & & \\
\hline & Plan Catedrales & & & & & & & & & & & & & & & & & & & & & & & \\
\hline & Rehabimed & & & & & & & & & & & & & & & & & & & & & & & \\
\hline & Recopar & & & & & & & & & & & & & & & & & & & & & & & \\
\hline & Síntesis propuesta & & & & & & & & & & & & & & & & & & & & & & & \\
\hline
\end{tabular}

\footnotetext{
${ }^{2}$ Los expertos reúnen las siguientes características: dos Catedráticos de Universidad en Rehabilitación y Patología, un Empresario de empresa constructora de rehabilitación de edificios de ámbito nacional, un Director de laboratorio acreditado de materiales de construcción, una Restauradora, un Arquitecto, un Arquitecto técnico y un Arqueólogo, todos ellos con reconocida experiencia profesional de más de veinte años. Dos Jefes de parque de bomberos uno de Madrid y otro de Sevilla, un Jefe de conservación de edificios de un Ayuntamiento de capital de provincia de 700.000 habitantes, un Director de conservación de edificio Patrimonio Mundial, un Jefe de conservación de autoridad portuaria, un Director de empresa de tasación de siniestros de ámbito internacional y un Experto en gestión de la calidad en edificación con numerosas publicaciones sobre el tema.
} 
Tabla 2.

Valoración de los expertos.

\begin{tabular}{|c|c|c|}
\hline $\begin{array}{c}\mathbf{N}^{\mathbf{o}} \\
\text { Factor }\end{array}$ & $\begin{array}{l}\text { Ordenación de factores } \\
\text { por importancia según } \\
\text { los expertos }\end{array}$ & $\begin{array}{l}\text { Nivel de incidencia del } \\
\text { factor en la durabilidad } \\
\text { Valor medio }\end{array}$ \\
\hline 5 & a. Conservación & 8,64 \\
\hline 10 & b. Seguridad incendio & 8,29 \\
\hline 6 & b. Modificación estado de cargas & 8,00 \\
\hline 2 & a. Diseño cubierta & 8,00 \\
\hline 9 & b. Instalaciones & 7,43 \\
\hline 15 & d. Referente patrimonial & 7,11 \\
\hline 17 & d. Actividad pastoral & 7,09 \\
\hline 1 & a. Situación geológica & 6,92 \\
\hline 4 & a. Sistema Constructivo & 6,83 \\
\hline 12 & c. Intensidad pluviométrica & 6,62 \\
\hline 7 & b. Sobre carga de uso & 6,54 \\
\hline 14 & d. Valor incremento población & 6,38 \\
\hline 13 & c. Variaciones de temperatura & 6,38 \\
\hline 8 & b. Ventilación & 6,29 \\
\hline 3 & a. Condiciones de entorno & 6,27 \\
\hline 16 & d. Valor mueble & 6,25 \\
\hline 11 & b. Medio ambiente interior & 5,86 \\
\hline
\end{tabular}

un alto grado de incertidumbre, el enfoque del método científico clásico, necesitaría obtener muchos datos de diverso tipo, físicos o estadísticos, costosos de obtener y que muchas veces no amortizarían su inversión. En estos casos se suele acudir a la opinión de expertos, sobre la materia que se trate y aceptar su conclusión.

Es por ello que nos hemos planteado utilizar otra herramienta matemática diferente, que no se ha aplicado hasta el momento, en este campo de los modelos de predicción de vida útil de los edificios. Nos referimos a la lógica difusa, cuyos principios básicos son:
- Se trata de una metodología matemática que representa el conocimiento impreciso donde la incertidumbre está presente.

- Nos permite aplicar la experiencia de expertos para imitar el razonamiento humano.

Como casi siempre existe una alternativa razonable entre la estricta lógica y la opinión de un experto, que nos permite establecer modelos de «razonamiento aproximado» o «sistemas expertos» con una base matemática que lo sustenta. El profesor Lofti A. Zadeh en 1965 planteo la teoría de conjuntos «borrosos» 0 «difusos» Fuzzy set, en la que se utiliza

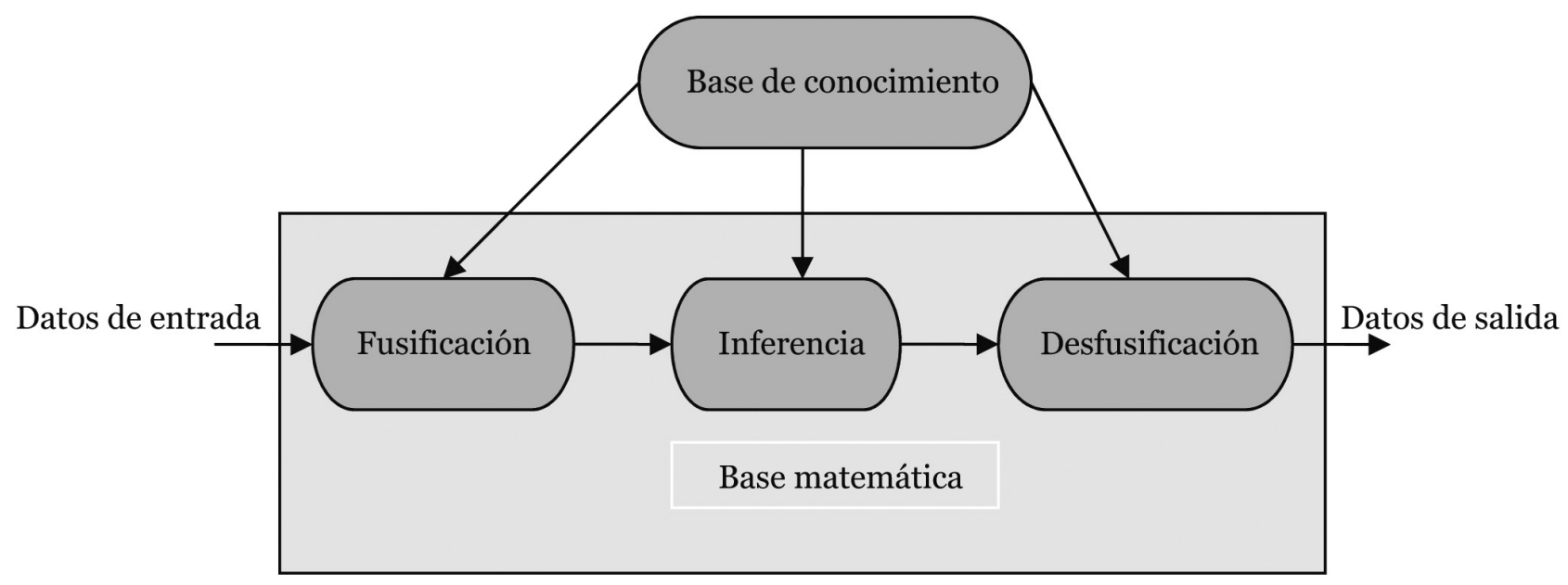

Figura 2. Esquema de de funcionamiento de un sistema difuso. 
el siguiente esquema de proceso matemático para imitar el razonamiento humano.

El esquema de funcionamiento de un sistema difuso lo mostramos en la Figura 2.

Como datos de entrada en la Figura 2: Partimos de unas variables lingüísticas de entrada que define y valora la persona con su lenguaje habitual, expresiones del tipo «hace calor»o «el edificio está bien conservado».

En el siguiente paso entramos en el mundo de los expertos y su base de conocimiento. El primer paso es el denominado fusificación, consiste en transformar las variables lingüísticas en funciones matemáticas, que llamaremos funciones de pertenencia. Para ello agrupamos las distintas expresiones en conjuntos dentro de un universo de discurso. Proponemos un ejemplo de fácil comprensión; Si hablamos de la altura de las personas (Universo de discurso) iría desde 1,50 m las más bajas, hasta 2,00 m las más altas y 1,75 la altura media o normal, por tanto una persona de 1,77 de altura estaría con un grado de pertenencia muy próximo a la media y a la vez otro grado de pertenencia menor a los mas altos. La función de pertenencia de un conjunto nos indica el grado en que cada elemento de un universo dado, pertenece a dicho conjunto.

\section{Fase de fusificación: variables de entrada y funcio- nes de pertenencia:}

La metodología se basa en determinar en primer lugar, variables de entrada que necesitan a su vez de la definición de una serie de funciones de pertenencia; en nuestro caso las variables de entrada son los diecisiete factores que ejercen influencia sobre la vida útil del edificio, que generarán sesenta y ocho funciones de pertenencia, cuatro funciones por cada factor, cada función nos proporcionará a su vez unos resultados de salidas. Posteriormente realizaremos una estructura de tipo jerárquico, que nos crean nuevas variables y nos obligará a determinar nuevas funciones de pertenencia, que se explicarán en su momento, con objeto de manejar un número menor de funciones y hacer más operativa la introducción de las reglas.

Una función de pertenencia nos describe el grado de aptitud o de funcionalidad de un factor principal. Un ejemplo puede ayudar a su comprensión: si analizamos el factor «Estado de conservación y adecuación al uso (Mantenimiento)», podríamos decir que el edificio presenta un grado de conservación (Condiciones de calidad): 1 óptimo; 2 normal; 3 degradado; 4 abandono. Las funciones de pertenencia nos permite conocer en qué grado participa de la condición de optimo, normal, degradado o abandono.

Para ilustrar como hemos procedido, exponemos brevemente las consideraciones para dos factores, uno que tiene un comportamiento excepcional no difuso y otro representativo del resto de factores difusos.

\section{Factor 1: Situación geológica}

El Instituto Geológico y Minero de España, establece cinco criterios de clasificación de condiciones constructivas en función del terreno que existe en cada zona. Se simplifica a cuatro estos criterios, ya que el primero, que es el óptimo, no se da en ningún caso en la provincia de Sevilla. (En el caso que el modelo se aplicara a otras áreas geográficas, habría que ajustar este factor a la realidad del entorno). Por tanto, el universo de discurso de este factor es: $1,2,3$, 4; donde el 1 corresponde a la mejor situación geológica posible y el 4 la peor situación posible.

Las funciones de pertenencia son de tipo lineal, dado que los valores que proporciona el Instituto Geológico y Minero del edificio, no son difusos.

\section{Factor 2: Diseño /Cubiertas}

La situación a la que debe responder el sistema experto para este factor es la siguiente:

Como ya hemos comentado, gran parte del deterioro del edificio se produce por la dificultad de evacuación de aguas en las cubiertas, que generalmente han sufrido modificaciones no sólo constructivas, sino fundamentalmente de tipo geométrico a lo largo del tiempo. La rapidez con la que el agua pueda evacuarse de la cubierta y la simplicidad de sus planos, afectan en gran medida a la vulnerabilidad del edificio.

Para estructurar nuestro modelo difuso hemos establecido cuatro funciones de pertenencia para este factor, cuyo universo de discurso está comprendido entre los valores adimensionales: 1 (la mejor situación posible hasta el valor) y 8 (la peor situación posible).

A la hora de establecer las funciones de pertenencia vamos a considerar una distribución Normal que entendemos se adapta mejor a nuestras necesidades. El motivo de elegir la «campana de Gauss», es debido a que la morfología de las cubiertas, tiende a asociarse a situaciones medio ambientales que suelen describirse con este tipo de distribución.

En esta fase, se ha contado con la opinión de los expertos, para establecer las funciones de pertenencia.

\section{Reglas fuzzy}

Este apartado es probablemente el de mayor incidencia en el resultado final, una buena base de reglas nos garantiza un buen funcionamiento del sistema.

Las reglas las podemos enunciar de la siguiente manera:

Si: «el agua de cubierta se evacua con rapidez» y «el sistema constructivo es adecuado».

Entonces: «la durabilidad es alta».

Aplicando fuzzy logic en la evaluación de reglas, todas las reglas cuyas premisas tengan algún grado de veracidad, influirán también en cierto grado a la solución del problema. Es decir toda regla que algo sepa, algo opina.

Para concretar las reglas entre las distintas variables de nuestra propuesta, se ha solicitado el concurso de los expertos.

Mostramos a continuación un ejemplo del resultado de respuestas a las reglas planteadas.

Factores de peligrosidad: antrópicos.

Regla 31.- factores de peligrosidad: Antrópicos.

Si: Valor incremento de población: regular.

Y Valor histórico artístico: regular.

Y Valor mueble: regular.

Y Nivel ocupación: bueno.

Entonces: el riesgo será: (ver Figura 3) 


\begin{tabular}{|l|c|c|c|c|c|}
\cline { 2 - 6 } \multicolumn{1}{c|}{} & Buena & Regular & Malo & Muy malo & NS/NC \\
\hline Riesgo & 2 & 7 & $\mathbf{1}$ & $\mathbf{0}$ & 1 \\
\hline
\end{tabular}

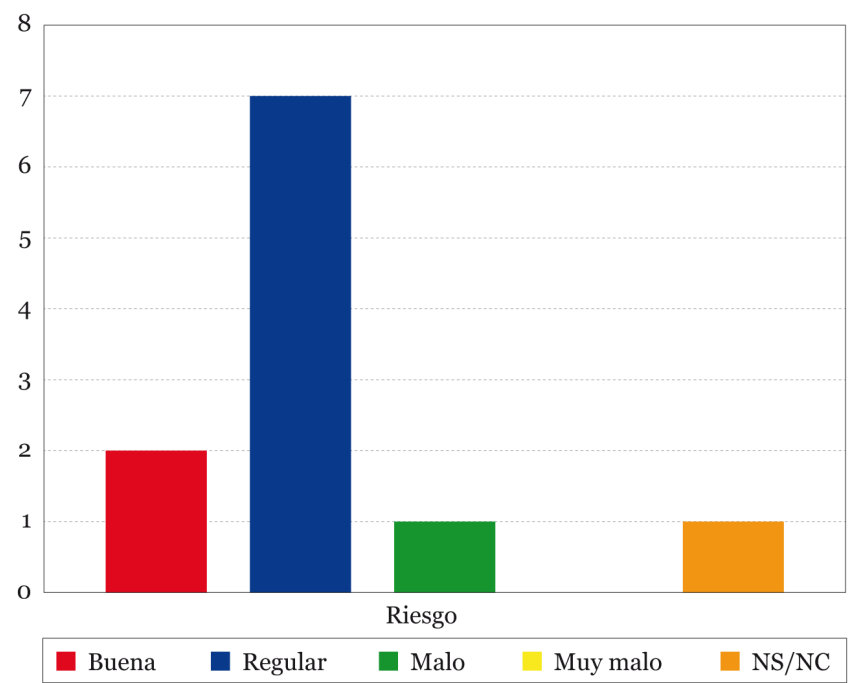

Figura 3. Resultados de la opinión de los expertos para la regla sobre riesgo Antrópico.

$\mathrm{Al}$ analizar los datos obtenidos, en este apartado, de las valoraciones de cada regla, por cada uno de los expertos, observamos, cómo cada resultado nos muestra en sí mismo un conjunto difuso.

\section{Estructura jerárquica planteada en el modelo}

Este paso, en el desarrollo del modelo, es de vital importancia, ya que al establecer una jerarquía hacemos prevalecer unos factores sobre otros, y condicionar fuertemente el resultado final. En la actualidad seguimos profundizando en esta cuestión, no obstante y con los datos obtenidos hasta el momento se formula una propuesta, que entendemos se encuentra en la dirección correcta.

$\mathrm{Al}$ estudiar detenidamente los resultados obtenidos en el apartado anterior referente a las reglas de pertenencia y teniendo en cuenta la opinión de los expertos, en sus comentarios a la incidencia de cada factor en el resultado final, véase Tabla 2, concluimos que:

1. Los factores de conservación y el de diseño de cubierta tienen un mayor peso que los otros tres factores de vulnerabilidad (Situación geológica, Condiciones de entorno y Sistema constructivo).

2. Los factores de riesgo antrópico están directamente relacionados con el estado de conservación.

3. Las modificaciones en el estado de cargas, y el estado de las instalaciones también están relacionadas con el estado de conservación.

4. Los riesgos atmosféricos inciden directamente en la durabilidad del conjunto.

Estas cuatro conclusiones, consecuencia de los datos e información aportados por los expertos, nos invita a que planteemos el siguiente esquema de relaciones jerárquicas entre los factores y las reglas de pertenencia establecidas.

En la Tabla 3, se expresa lo enunciado anteriormente.
Esta estructura jerárquica nos permite trabajar con un número reducido de variables en cada nivel, consiguiendo así una mejor operatividad. Establecemos cuatro grupos de reglas, que las denominamos con la siguiente terminología:

Reglas «A» o Reglas directas

Reglas «B» o reglas de segundo nivel subordinadas

Reglas «C» o reglas de tercer nivel subordinada y directa

Explicamos a continuación las Reglas «A» o Reglas directas que nos sirven para ilustrar la forma de operar con el resto de reglas. Las de tipo «A» son aquéllas que se aplican directamente sobre cada agrupación de los factores principales, y que tienen como consecuencia las siguientes variables de segundo nivel, a las que denominamos: Vulnerabilidad 1, Vulnerabilidad 2, Riesgo estructural 1, Riesgo estructural 2, Riesgo atmosférico, y Riesgo antrópico. A modo de ejemplo, obsérvese que la variable Vulnerabilidad 1 , viene dada por los resultados de los valores aplicados a los factores denominados Cubierta y Conservación. Para ella se han definido cinco funciones de pertenencias siguiendo la tipología de campana de Gaus, con un universo de discurso, que va de cero a diez, con objeto de ampliar la escala de los resultados.

\section{Fase de desfusificación}

Para la última fase de este proceso hemos utilizado el método clásico de desfusificación del centro de área, que nos aporta el valor medio del conjunto difuso resultante de todo el proceso anterior, y que se convierte en la variable de salida, en nuestro caso los Años de vida útil estimada.

Una vez construido el modelo, lo hemos implementado utilizando el programa informático XFUZZY 3.0 (10), dando, lugar al: Fuzzy building service life (FBSL) que nos posibilita obtener un valor de la vida útil en años, del edificio.

Esta herramienta informática nos permite introducir todos los datos que hemos obtenido del medio centenar de edificios analizados, utilizando la ficha de toma de datos expuesta en la Figura 4, en la que viene indicado el entorno de «valoraciones difusas». Asignamos valor uno a la mejor situación posible para cada factor y cuatro a la peor, permitiendo que se expresen valores intermedios, según la situación real de cada factor. La ficha propuesta ha de ser completada por un experto en conservación de edificios, introduciendo además los siguientes datos: nombre del inmueble, situación y municipio, fecha de la toma de datos, número de registro según la Archidiócesis de Sevilla y número de ficha del estudio. También se aportan planos de planta y alzado reducidos, el plano catastral está orientado al norte. Se utilizan algunas fotografías tomadas durante la inspección. El objetivo de estos planos e imágenes es situarnos espacialmente en el lugar concreto que estudiamos.

Como puede comprobarse, la ficha, además de los datos gráficos del edificio, incorpora los factores del inmueble que pueden afectar su durabilidad y vulnerabilidad a partir de su análisis arquitectónico, del entorno en el que se ubica y de los aspectos constructivos. Considera además su estado de conservación y los valores de peligrosidad estático-estructural y antrópicos.

Cuando se tienen todos los datos de cada edificio, estos son introducidos en el programa, el cual contiene una aplicación que posibilita implementar las instrucciones para que interrelacionen las variables con su nivel ponderado. La Figura 5, muestra la pantalla del programa informático XFUZZY 3.0 
Tabla 3.

Relaciones jerárquicas entre los factores.

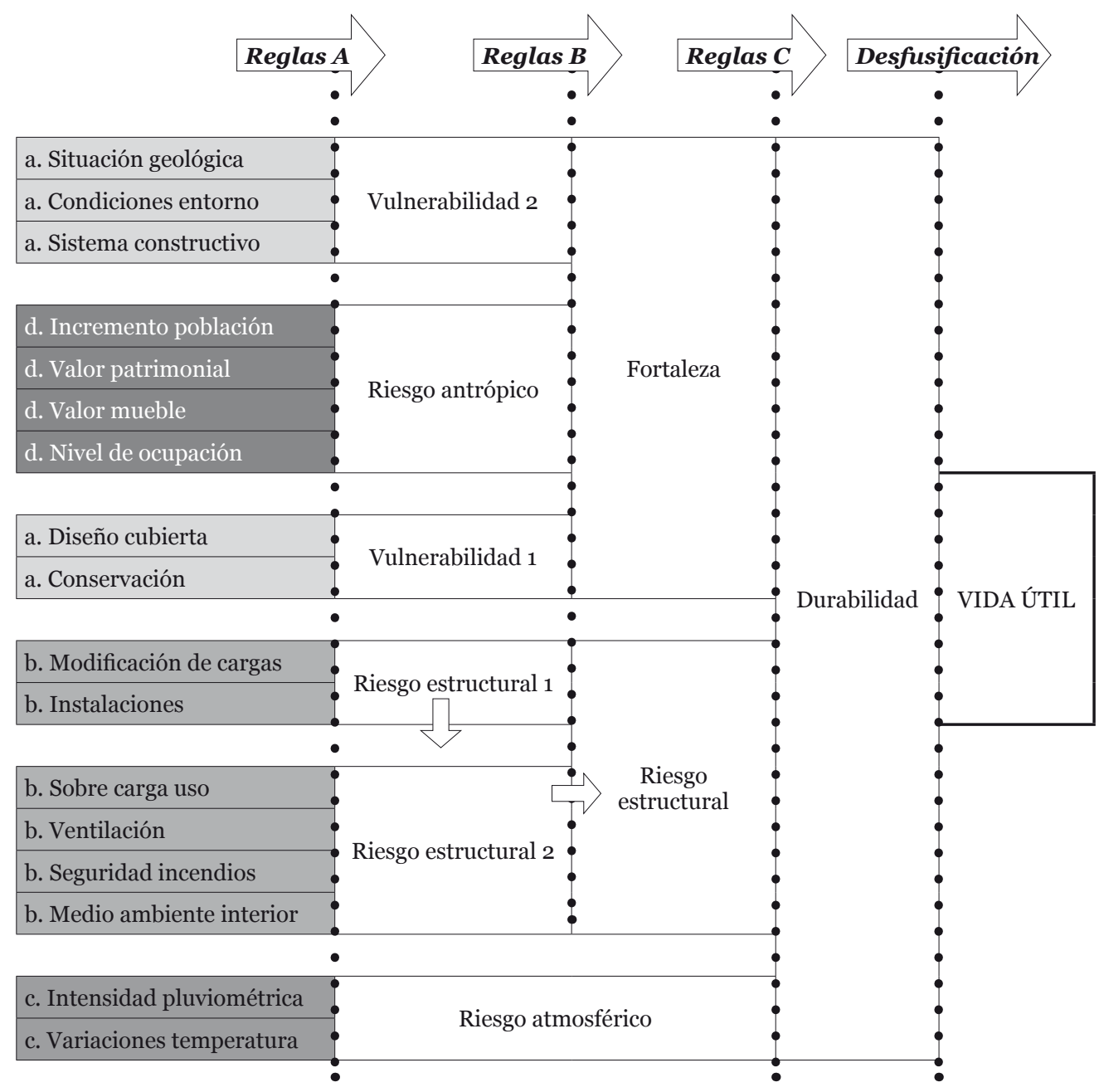

(11), con la implementación del esquema de relaciones jerárquicas que presentamos en la Tabla 3.

\subsection{Fase $4^{\text {a }}$ Ordenar el conjunto de edificios según su vida útil estimada.}

Una vez que el Modelo realiza el tratamiento de los datos que le hemos introducido, nos proporciona, en valor numérico, la vida útil de los inmuebles en años, por lo que podemos proceder a su ordenación. En la Tabla 4, se ha reflejado la relación de los 50 edificios, a los que se le ha aplicado el Fuzzy building service life (FBSL), ordenados de menor a mayor según la estimación de su vida útil.

\section{VALIDACIÓN DEL MODELO}

El modelo pretende ser un indicador de tendencias de evolución del edificio en el futuro, por ello nos interesa comprobar, al menos de manera inicial, su validez. Para ello hemos utilizado tres vías que nos han permitido realizar comparaciones entre diversos sistemas de predicción.

En primer lugar, en un trabajo previo que fue la primera aportación que se realizó (12), ya teníamos propuesta una tabla de predicción de durabilidad realizada a partir de datos técnicos cuyas conclusiones fueron las siguientes:
Comparamos «durabilidad» con «vulnerabilidad y el riesgo», para concluir que la siguiente formulación se ajusta a estas relaciones:

Durabilidad $=1 / \mid($ Vulnerabilidad - Vulnerabilidad $\mathrm{x}$ Riesgo $) \mid$

La Durabilidad es el resultado de valorar la vulnerabilidad y el riesgo entre los valores de uno a cuatro como ya hemos indicado. Por tanto obtenemos valores de 1,00 para una durabilidad muy favorable y de 0,08 para una durabilidad muy desfavorable.

Analizando los resultados obtenidos, constatamos lo siguiente:

$1^{\mathrm{o}}$ Cuando el riesgo disminuye la durabilidad tiende a infinito, parece lógico ya que el bien patrimonial si no estuviera sometido a ningún riesgo su durabilidad aumentaría, es lo que pretenden los museos conservando las obras de arte. Así mismo cuando el riesgo aumenta la durabilidad disminuye más rápidamente.

$2^{\mathrm{O}}$ Para situaciones de riesgo entre el valor 1 y 2 (situaciones de riesgo bajo) es donde las variaciones con respecto a la durabilidad son mayores, también parece lógico este resultado: si las situaciones de riesgo son bajas, la durabilidad es alta. 
Estudiando el entorno de valores obtenidos de los riesgos en función de la vulnerabilidad y la durabilidad constatamos lo siguiente:

$1^{\mathrm{o}}$ La formula nos indica que cuando la vulnerabilidad disminuye la durabilidad aumenta, no hasta el infinito como ocurría antes, sino hasta un valor concreto donde la gráfi-

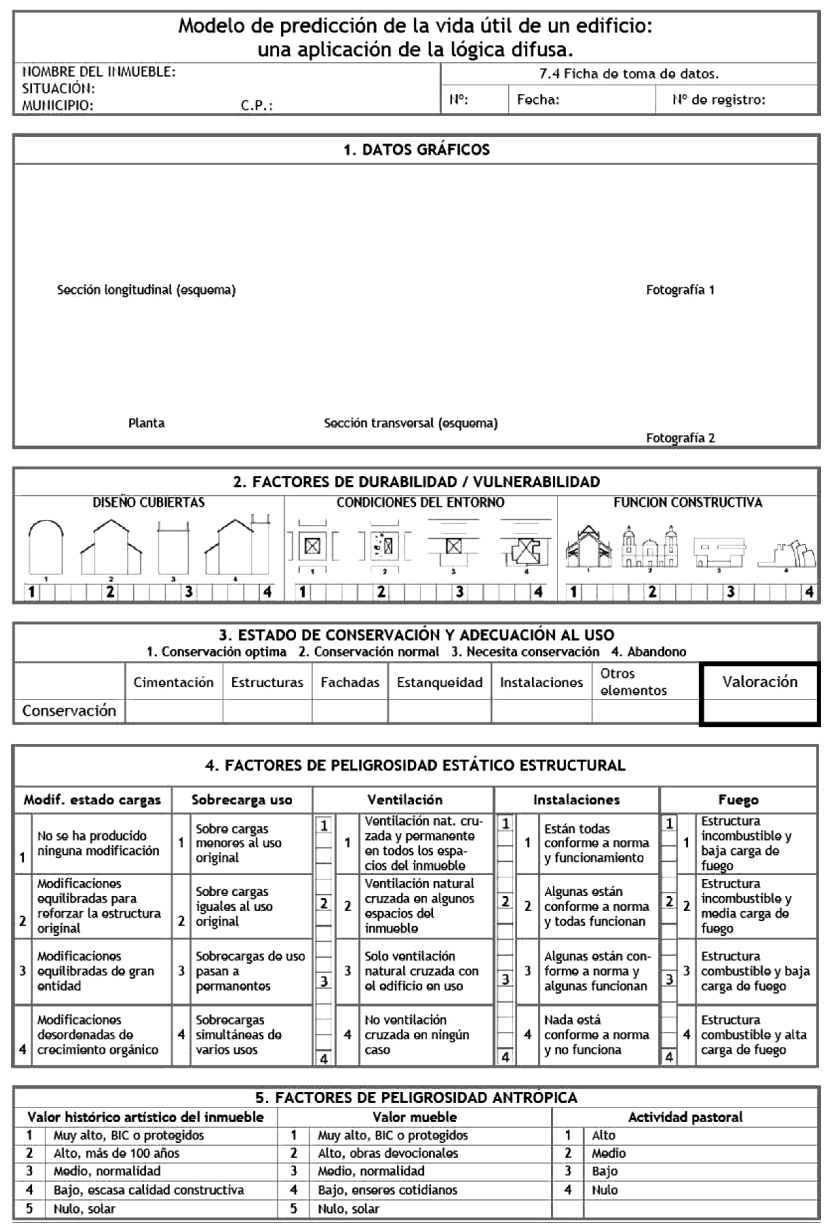

Figura 4. Ficha de toma de datos. ca interseca con el valor 1 de mejor vulnerabilidad posible. Parece lógico ya que valores de vulnerabilidad por debajo de uno corresponderían a edificios perfectos, y esto no se da nunca en arquitectura.

$2^{\mathrm{o}}$ Para vulnerabilidad alta la durabilidad no decrece bruscamente, también parecía lógico ya que los edificios se realizan con estándares constructivos similares y porque el mantenimiento es el que garantiza ese nivel óptimo o no de vulnerabilidad.

Como primer resultado de todo el proceso, obtuvimos una clasificación ordenada de los edificios según la necesidad de intervención sobre el bien, aplicando el criterio que eligiéramos de clasificación (por situaciones de riesgo, vulnerabilidad, peligro, situación geográfica, etc.)

Los resultados obtenidos, aplicados a los cincuenta edificios objeto de estudio, los hemos comparado con los resultados obtenidos con el nuevo modelo FBSL. Para ello se ha utilizando el coeficiente de correlación de Pearson, obteniendo un valor de 0,65 , lo que interpretamos como que, aunque siendo dos metodologías diferentes, ambas apuntan en una misma dirección, lo que nos da cierta fiabilidad.

En segundo lugar, se le ha pedido a dos expertos que aportaran su opinión en relación a quince de los edificios estudiados y se han comparado sus respuestas con los datos obtenidos con el modelo FBSL. En este caso la valoración estimada de vida útil realizada por los profesionales, a partir de la media aritmética entre los datos aportados por cada uno y los valores obtenidos por nuestro modelo, muestran gran afinidad, ya que se obtiene una correlación de 0,90, considerada muy alta.

En tercer lugar, y dado que el Modelo de la norma ISO 156861 , es el único de los métodos que hemos encontrado en la literatura, que analiza la vida útil de un edificio, a partir de los criterios propuestos por el Politécnico de Milán y de la utilización del método de Montecarlo33, la hemos aplicado a los 50 edificios de la Archidiócesis de Sevilla. Los resultados obtenidos por ambos modelos no muestran correlación alguna. A nuestro entender, los motivos de esta diferencia podrían ser los siguientes:

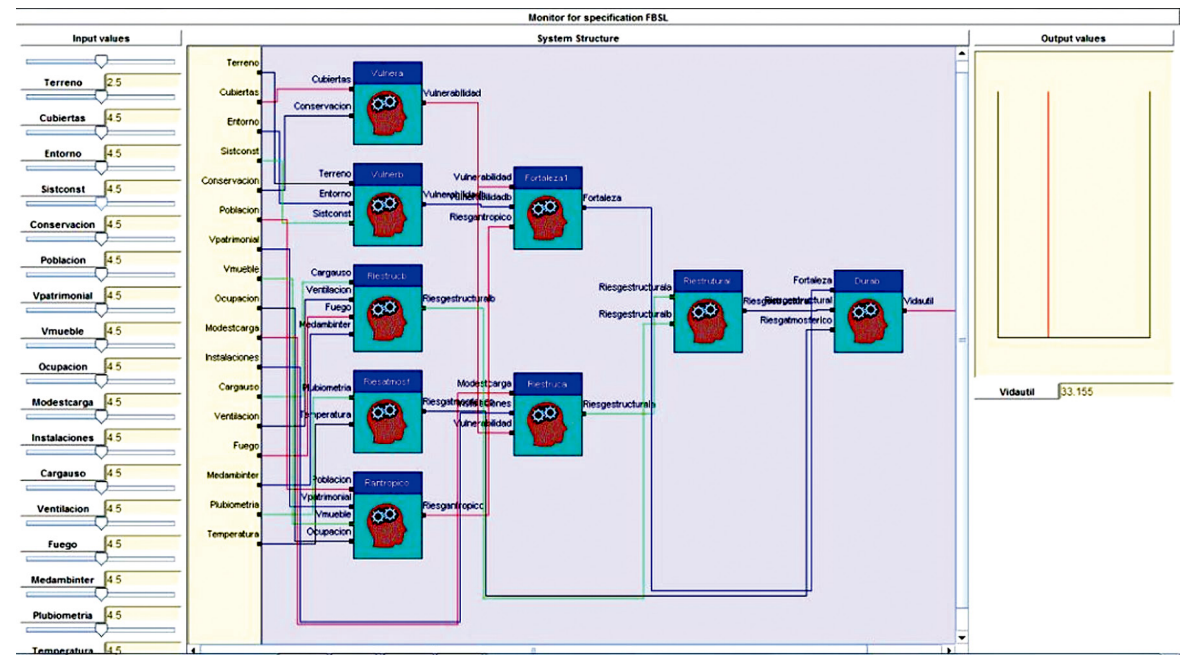

Figura 5. Pantalla que muestra un ejemplo de interrelación de cada variable con el programa XFUZZY 3.o.

\footnotetext{
${ }^{3}$ El método de Montecarlo es un método no determinístico o estadístico numérico, usado para aproximar expresiones matemáticas complejas y costosas de evaluar con exactitud.
} 
Tabla 4.

Ordenación de los edificios según su vida útil estimada.

\begin{tabular}{|c|c|c|c|}
\hline $\begin{array}{l}\text { FECHA ultima } \\
\text { actualización }\end{array}$ & NOMBRE EDIFICIO & POBLACION & $\begin{array}{l}\text { SEGÚN MODELO } \\
\text { FBSL en años }\end{array}$ \\
\hline 2-7-09 & CASA RECTORAL & PRUNA & 12 \\
\hline $19-1-10$ & NUESTRA SEÑORA DE LA ASUNCIÓN & LORA DEL RÍO & 14 \\
\hline $23-6-09$ & LOCALES CESIÓN & ROSALES (LOS) & 15 \\
\hline $29-12-10$ & PARROQUIA DE SAN FERNANDO & VILLANUEVA DEL RÍO Y MINAS & 16 \\
\hline 5-5-09 & CAPILLA SAN LORENZO & CAMPANA (LA) & 17 \\
\hline $20-12-10$ & CASAS RECTORAL & SANTIPONCE & 17 \\
\hline $1-6-10$ & SAN PABLO CASA RECTORAL & TRAJANO & 18 \\
\hline 11-4-09 & SANTA MARÍA DE GRACIA & ALMADÉN DE LA PLATA & 18 \\
\hline $3-2-10$ & CONVENTO DEL ESPÍRITU SANTO & GUADALCANAL & 19 \\
\hline $7-5-09$ & IGLESIA CONVENTUAL SAN JOSÉ & FUENTES DE ANDALUCÍA & 19 \\
\hline $3-2-10$ & PARROQUIA SAN SEBASTIÁN Y SAN DIEGO & SAN NICOLÁS DEL PUERTO & 20 \\
\hline $25-5-10$ & PARROQUIA SAN JUAN BAUTISTA & ALCOLEA DEL RÍO & 20 \\
\hline $19-1-10$ & PARROQUIA SAN MIGUEL & LORA DE ESTEPA & 21 \\
\hline $24-6-09$ & PARROQUIA SAN BENITO & SEVILLA & 21 \\
\hline $19-1-10$ & CONVENTO DEL ESPÍRITU SANTO & GUADALCANAL & 21 \\
\hline 19-1-10 & PARROQUIA SANTIAGO EL MAYOR & HERRERA & 21 \\
\hline $17-4-09$ & CASA RECTORAL & RONQUILLO (EL) & 22 \\
\hline $3-1-11$ & PARROQUIA SANTA MARTA & MOLARES (LOS) & 22 \\
\hline $17-4-09$ & ERMITA NUESTRA SEÑORA DE GRACIA & RONQUILLO (EL) & 24 \\
\hline 23-6-09 & COCHERA CASA RECTORAL & ROSALES (LOS) & 24 \\
\hline $11-1-11$ & PARROQUIA NTRA SÑRA DE LA VICTORIA & OSUNA & 25 \\
\hline $25-5-10$ & PARROQUIA SAN JUAN BAUTISTA & ALCOLEA DEL RÍO & 27 \\
\hline 24-6-09 & PARROQUIA SAN BERNARDO & SEVILLA & 27 \\
\hline $1-6-10$ & PARROQUIA SAN PABLO & TRAJANO & 28 \\
\hline $24-11-10$ & PARROQUIA SANTA MARIA DE LAS NIEVES & ALANÍS & 28 \\
\hline 6-11-09 & PARROQUIA NTRA. SRA. DE LA PAZ & MARINALEDA (MATARREDONDA) & 28 \\
\hline $27-1-11$ & PARROQUIA PURISIMA CONCEPCIÓN & NAVAS DE LA CONCEPCIÓN (LAS) & 29 \\
\hline $1-6-10$ & CAPILLA DE LA TRINIDAD & LEBRIJA & 29 \\
\hline $19-1-10$ & PARROQUIA SAN ISIDRO & PRIORATO (EL) & 30 \\
\hline $3-1-11$ & PARROQUIA SAN JOSÉ & MORÓN DE LA FRONTERA & 30 \\
\hline $11-1-11$ & COLEGIATA DE OSUNA & OSUNA & 30 \\
\hline 20-4-09 & PARROQUIA DIVINO SALVADOR & CASTILLEJA DE LA CUESTA & 30 \\
\hline $23-6-09$ & PARROQUIA NUESTRA SEÑORA DE FÁTIMA & ROSALES (LOS) & 31 \\
\hline $15-6-10$ & CASA RECTORAL & ARAHAL & 33 \\
\hline 2-7-09 & PARROQUIA SAN ANTONIO ABAD & PRUNA & 33 \\
\hline 11-4-10 & PARROQUIA SAN BARTOLOMÉ & REAL DE LA JARA (EL) & 34 \\
\hline $25-5-09$ & PARROQUIA SAN ANDRÉS Y SAN MARTÍN & SEVILLA & 35 \\
\hline 29-6-09 & COMPLEJO PARROQUIAL PINZÓN & PINZÓN & 36 \\
\hline $20-4-10$ & NUESTRA SEÑORA DE BELÉN & TOMARES & 36 \\
\hline 10-6-09 & PARROQUIA SAN MARCOS & SEVILLA & 36 \\
\hline $10-6-09$ & SAN ROMAN Y SANTA CATALINA & SEVILLA & 38 \\
\hline $24-6-09$ & PARROQUIA SAN SEBASTIÁN & SEVILLA & 38 \\
\hline $24-11-10$ & SANTA MARÍA DEL PUEBLO DE DIOS & SEVILLA & 41 \\
\hline $10-6-09$ & PARROQUIA SAN ROQUE & SEVILLA & 42 \\
\hline $18-6-09$ & PARROQUIA SAN LORENZO & SEVILLA & 42 \\
\hline $3-2-10$ & IGLESIA DE SANTA ANA & GUADALCANAL & 43 \\
\hline $10-2-11$ & PARROQUIA SAN JOAQUÍN & SEVILLA & 43 \\
\hline $17-4-09$ & PARROQUIA DIVINO SALVADOR & RONQUILLO (EL) & 43 \\
\hline $20-12-10$ & PARROQUIA SAN ROQUE & CABEZAS DE SAN JUAN (LAS) & 45 \\
\hline $23-6-09$ & SALONES PARROQUIALES & ROSALES (LOS) & 52 \\
\hline
\end{tabular}


La norma ISO proporciona resultados basados en la probabilidad estadística. Es decir, indica que la probabilidad más alta está en una fecha determinada, pero además se fija con una probabilidad que está entre los porcentajes del $25 \%$ y el $30 \%$, en relación a la referencia de vida útil estimada en el proyecto. (RLS).

1. El método de Montecarlo, supone la selección del tipo de distribución de cada factor considerado, con lo que la falta de correlación observada puede deberse a la forma de aplicación del método y no al método en sí mismo.

2. Con la norma ISO no se tiene constancia que se hayan realizado análisis aplicados a edificios completos, ya que se trata de un modelo que está orientado a elementos constructivos, no a sistemas constructivos y aún menos al edificio en su conjunto.

3. La norma ISO parte de la premisa de la estimación a nivel de proyecto de la referencia de la vida de servicio (RSL). En nuestro caso, los edificios estudiados son en su mayor parte muy antiguos, por lo que no se dispone del proyecto arquitectónico y en consecuencia tampoco de la vida útil de servicio que se estimó para el edificio.

\section{CONCLUSIONES}

Tras el proceso de diseño del Modelo FBSL, la validación y su aplicación en cincuenta edificios objeto del estudio, comprobamos que permite las siguientes acciones:
$1^{0}$ Interrelacionar factores que afectan a la evolución en el tiempo de los edificios y ponderar su incidencia.

$2^{\mathrm{O}}$ Establecer una estructura jerárquica de relaciones entre los factores, y realizar los ajustes oportunos en función de los datos de monitorización del edificio que realmente se dispongan.

$3^{0}$ Medir, de forma parcial, la incidencia de cada factor, lo que nos permite saber en que factor actuar y su efecto en el resultado final.

$4^{\mathrm{o}}$ Corrige la incertidumbre inherente a la opinión de los expertos, lo que aporta objetividad a los resultados obtenidos.

$5^{\circ}$ Proporciona valores de la vida útil en años (en una escala de uno a cien años, en función de una durabilidad: 1 muy alta, 2 alta, 3 baja, 4 muy baja), en un entorno de fiabilidad razonable.

$6^{\circ}$ En general sus resultados son validos si son comparados con muestras amplias de edificios.

Sólo cabe añadir que en caso de que se decida aplicar este modelo de valoración de la vida útil a un conjunto de edificaciones patrimoniales con características diferentes al estudiado, será necesaria una revisión de los criterios de ponderación. La causa principal, es que al cambiar el entorno son necesarios ajustes debidos a factores que interaccionan con: la tipología, los usos y, lógicamente los emplazamientos específicos de los inmuebles.

\section{REFERENCIAS}

(1) Chávez-Hernández, J. A., Recarey, C. A., García-Lorenzo, M. M., López-Jiménez, O. (2012). Utilización de la Inteligencia Artificial en el diagnostico patológico de edificaciones de valor patrimonial. Informes de la Construcción, 64(527): 297305, doi: http://dx.doi.org/10.3989/ic.11.036.

(2) Zadeh, L. (1965). Fuzzy Sets. Information and Control, 8(3): 338-353, doi: http://dx.doi.org/10.1016/Soo199958(65)90241-X.

(3) Kosko, B. (2010). El futuro borroso o el cielo en un chip. p. 448, Barcelona. Editorial Crítica.

(4) A.A.V.V. (1987). Carta del Rischio. Italia. Istituto Superiore per la Conservazione ed il Restauro.

(5) Monjo, J. (2007). Durabilidad vs vulnerabilidad. Informes de la Construcción, 59(507): 43-58, doi: http://dx.doi. org/10.3989/ic.2007.v59.i507.531.

(6) ISO, Norma ISO 15686 Standards on Service Life Planing 2011.

(7) Cardona, O. D. (2001, 29 y 30 de junio). La necesidad de repensar de manera holística los conceptos de vulnerabilidad y riesgo: «Una crítica y una revisión necesaria para la gestión». Artículo y ponencia para International Work-Conference on vulnerability in Disaster Theory and practice. Wageningen, Holanda. Disaster studies of Wageningen University and Research Center.

(8) AENOR, Norma UNE 41805 Diagnóstico de edificios. Madrid, 2009.

(9) Macías, J.M., Calama, J. Ma ., Chávez, Ma . J. (2012). Factores de vulnerabilidad y riesgo para determinar la vida útil de un edificio; bases para aplicar la lógica difusa. $4^{\circ}$ Congreso de patología y rehabilitación de edificios - PATORREB.

(10) AA.VV. (2012). $6^{\text {th }}$ International Seminar on Urban Conservation Measuring Heritage Conservation Performance Organized by Silvio Mendes Zancheti \& Katriina Similä (C) CECI \& ICCROM

(11) Instituto de Microelectrónica de Sevilla (IMSE-CNM). (2012). Herramientas de cad para lógica difusa: http://www2. imse-cnm.csic.es/Xfuzzy/Xfuzzy_3.o/download_sp.html. Sevilla.

(12) Macías, J.M. (2010). Proyecto Fin de Máster. Máster en Conservación y Restauración del Patrimonio Histórico. IAPH. Universidad de Sevilla. 